\title{
Hemangioendotelioma epitelioide hepático: un reto diagnóstico de los tumores vasculares
}

\author{
Hepatic epithelioid hemangioendothelioma: a diagnostic challenge of vascular tumors
}

Carlos Pacheco-Molina1, Armando A. Baeza-Zapata², Óscar R. García-De León', Josefina B. Leyva-Alamillo', Francisco Vásquez-Fernández y Marco A. Hernández-Guedea ${ }^{1}$

${ }^{1}$ Departamento de Cirugía General; ²Departamento de Gastroenterología y Endoscopia Digestiva. Hospital Universitario José Eleuterio González, Monterrey, Nuevo León, México

\section{Resumen}

El hemangioendotelioma epiteloide es un tumor muy infrecuente, con una presentación variable y un comportamiento clínico impredecible. No se han precisado la etiología ni los factores de riesgo desencadenantes. A diferencia de otros tumores hepáticos primarios, no surge en el contexto de una enfermedad crónica del hígado. El abordaje es un reto debido al espectro de posibilidades y la necesidad de inmunohistoquímica para establecer el diagnóstico definitivo. La información disponible hasta el momento es limitada por los escasos casos publicados, lo que favorece que las opciones terapéuticas sean pocas o no exista la evidencia suficiente para estandarizarlas cuando la lesión no sea resecable.

Palabras clave: Hemangioendotelioma epitelioide hepático. Tumores hepáticos. Resección hepatica.

\begin{abstract}
Epitheloid hemangioendothelioma is a very rare tumor, with a variable presentation and unpredictable clinical behavior. The etiology and the triggering risk factors have not been specified. Unlike other primary liver tumors, it does not arise in the background of chronic liver disease. The approach is challenging due to the spectrum of possibilities and the need for immunohistochemistry to establish the definitive diagnosis. The information available so far is limited due to the few published cases, this favors that the therapeutic options are few or that there is insufficient evidence to standardize them when the lesion is not resectable.
\end{abstract}

Key words: Hepatic epithelioid hemangioendothelioma. Liver malignancies. Liver resection.

\section{Introducción}

El hemangioendotelioma epitelioide es un tumor raro con una incidencia menor de 0.1 por 100,000 habitantes ${ }^{1}$, de origen endotelial y del tejido conecti$\mathrm{vo}^{2}$, con un potencial intermedio de malignidad ${ }^{3}$, de etiología desconocida y curso natural impredecible; su espectro clínico es considerado entre el hemangioma y el angiosarcoma'.

En 1975 fue descrito por Dail y Liebow con el nombre de tumor bronquioalveolar intravascular ${ }^{4,5}$; posteriormente, Corrin, et al. ${ }^{6}$ identificaron sus características

\section{Correspondencia:}

*Armando A. Baeza-Zapata

Avda. Madero y Gonzalitos s/n

Col. Mitras Centro

Fecha de recepción: 16-07-2020

C.P. 64460, Monterrey, N.L., México

E-mail: armando-baeza@outlook.com

D009-7411/0 2020 Academia Mexican (http://creativecommons.org/licenses/by-nc-nd/4.0/).
Fecha de aceptación: 16-12-2020

DOI: $10.24875 /$ CIRU.20000777
Cir Cir. 2021;89(S1):6-9

Contents available at PubMed www.cirugiaycirujanos.com ajo la licencia CC BY-NC-ND 
angiogénicas mediante la presencia de cuerpos de Weibel-Palade, y Weldon-Linne, et al. ${ }^{7}$ observaron la expresión en las células tumorales del antígeno relacionado con el factor VIII (FVIII-RAg) ${ }^{5}$. No fue hasta 1982 que Weiss y Enzinger ${ }^{8}$ hicieron la definición formal, y desde ese momento ha recibido diversos nombres y existen reportes que no exceden los 500 casos hasta la década pasada ${ }^{1,9}$.

\section{Caso clínico}

Varón de 64 años con antecedente de hábito tabáquico a razón de 5 paquetes/año, que es referido por historia de 6 meses de evolución de dolor abdominal en el hipocondrio derecho, asociado a saciedad temprana, fatiga y aumento del perímetro abdominal. Durante la exploración física se encuentra una masa dolorosa palpable en el hipocondrio derecho (Fig. 1). Por este motivo se realiza una tomografía computada de tórax y abdomen contrastada, que reporta una lesión hepática hipodensa de $16 \times 13 \times 21 \mathrm{~cm}$, con bordes mal definidos y realce nodular periférico discontinuo con relleno centrípeto progresivo que condicionaba compresión de la vena cava inferior, la vesícula biliar y el duodeno (Fig. 2).

Los marcadores tumorales (antígeno carcinoembrionario y alfa-fetoproteína) y las pruebas de funcionamiento hepático se encontraban dentro de los parámetros normales.

Se realizó biopsia con Trucut guiada por sonografía, que reportó por histopatología un hemangioma hepático. Se complementó con una resonancia magnética de abdomen, en la que se observó una lesión hiperintensa en secuencia T2 (Fig. 3), y con una tomografía por emisión de positrones que mostró la lesión con las características ya descritas (Fig. 4).

Al no evidenciarse enfermedad diseminada, se decidió tratamiento quirúrgico. Se realizó un abordaje subcostal bilateral, identificando una tumoración que ocupa y deforma el lóbulo hepático derecho (Fig. 5), con imposibilidad de movilización del lóbulo derecho por las dimensiones de esta. Se realizó disección de hilio hepático con ligadura de ramas derecha de la vena porta y la arteria hepática, maniobra de Hanging y transección del parénquima hepático con técnica de Kelly-clamp, utilizando dispositivo de energía y sellado. Se preservó la vena suprahepática media, la cual se encontraba desplazada en sentido medial con compresión extrínseca. Finalmente, se seccionó el conducto cístico y se extrajo la pieza quirúrgica

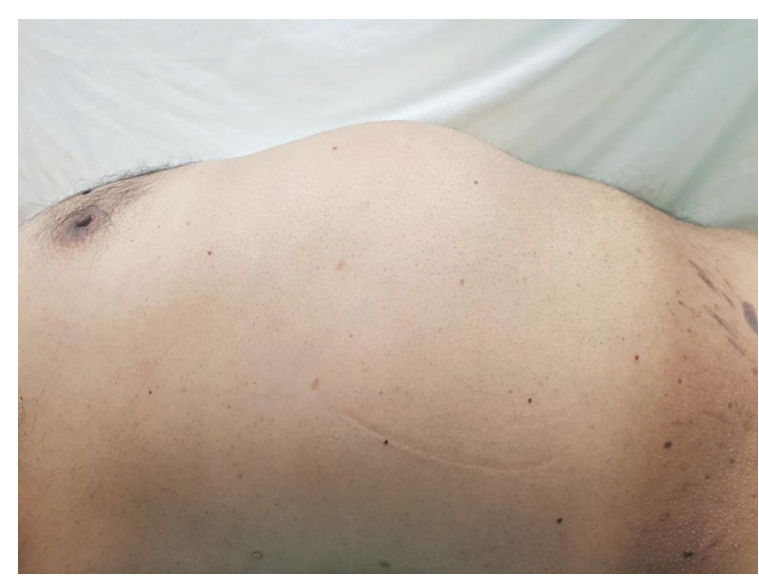

Figura 1. Elevación y asimetría en el hipocondrio derecho durante la exploración física, secundaria a una masa palpable dolorosa.

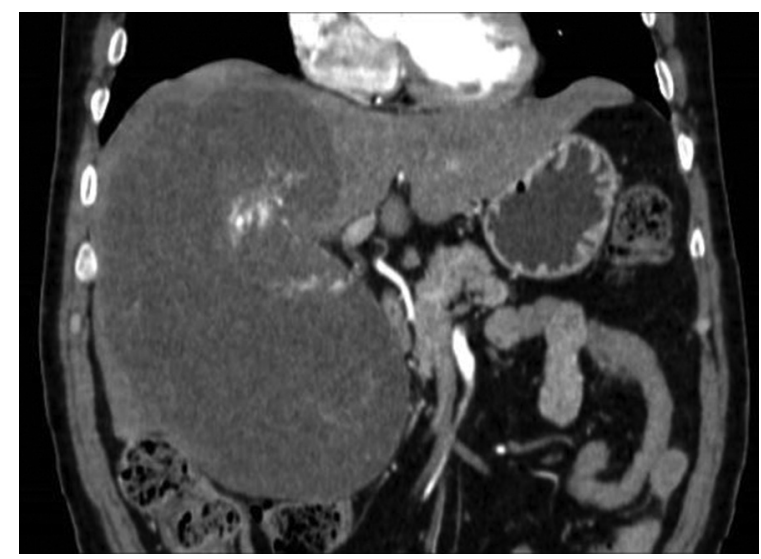

Figura 2. Corte coronal en tomografía computada de tórax y abdomen con contraste intravenoso que muestra una tumoración hipodensa irregular que desplaza las estructuras adyacentes.

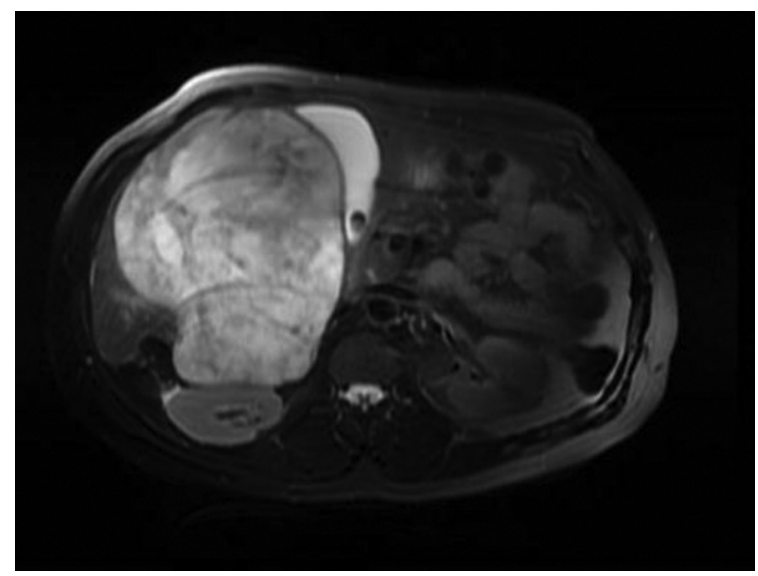

Figura 3. Corte axial de resonancia magnética abdominal en secuencia T2, que muestra una tumoración lobulada hiperintensa que deforma la anatomía hepática. 


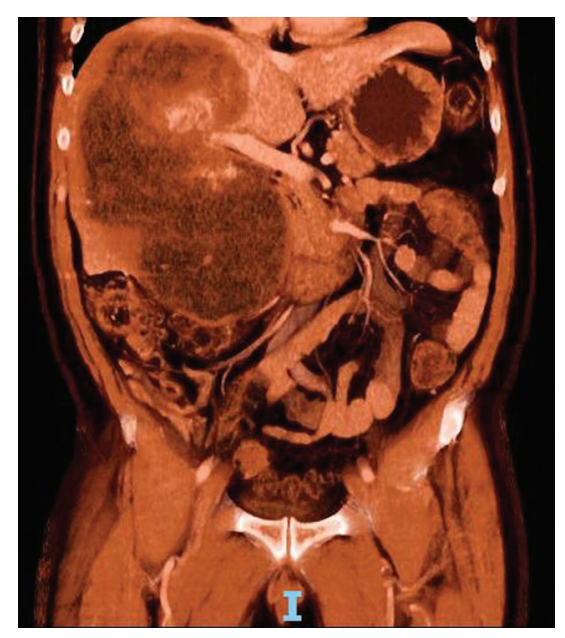

Figura 4. Corte coronal en tomografía por emisión de positrones que muestra una tumoración hepática lobulada que deforma la arquitectura desplazando las estructuras adyacentes.

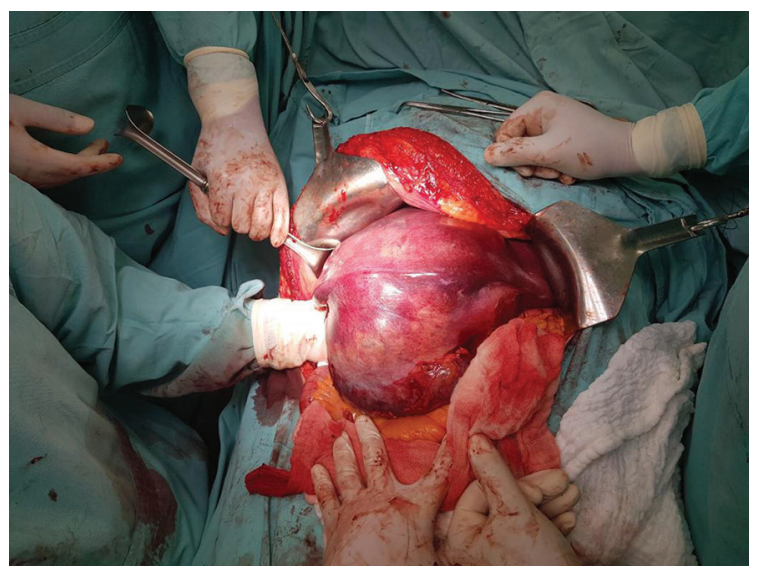

Figura 5. Exposición hepática durante el transoperatorio observando tumoración en lóbulo derecho.

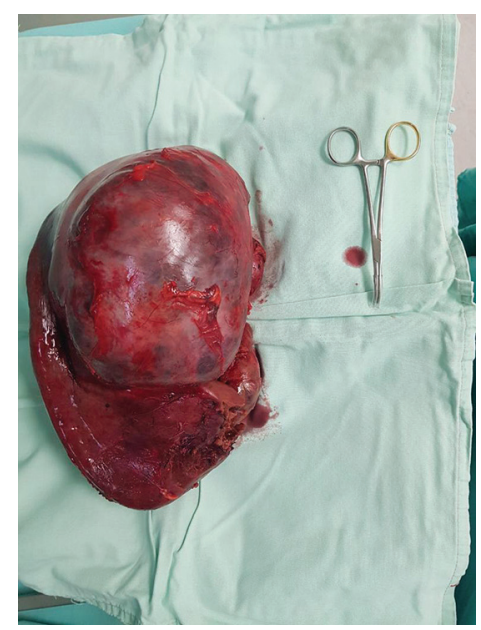

Figura 6. Producto de resección de hepatectomía derecha con tumoración sólida.
(Fig. 6), y se verificaron la hemostasia y la integridad de la vía biliar del remanente hepático.

El análisis histopatológico reportó tumoración epiteloide de variedad sólida, sin necrosis, con inmunohistoquímica positiva para CD34, CD31 y GLUT-1, de patrón focal, concluyente con hemangioendotelioma epitelioide con siete mitosis por diez campos de alto poder, y borde de resección quirúrgica libre de neoplasia a $1 \mathrm{~mm}$.

El paciente tuvo una evolución posquirúrgica inmediata adecuada, sin presencia de recurrencia ni metástasis extrahepáticas a 18 meses del procedimiento quirúrgico, y continúa en vigilancia por consulta externa.

\section{Discusión}

El hemangioendotelioma epitelioide es un tumor vascular distintivo de tejidos blandos que puede observarse en algunos órganos viscerales ${ }^{3}$. El órgano comúnmente afectado es el hígado, aunque se han descrito otras localizaciones, como los pulmones, el peritoneo, el bazo, el hueso, el cerebro, las meninges, la mama, el corazón, la cabeza y el estómago².

Varios factores se han considerado en su etiología, como anticonceptivos orales, cloruro de vinilo, asbestos, contraste Thorotrast, trauma mayor del hígado, hepatitis viral, colangitis biliar primaria, consumo excesivo de alcohol ${ }^{1}$ y algunas translocaciones cromosómicas específicas $t(1 ; 3)(p 36.3 ; q 25)$, que son típicas de este tumor ${ }^{2}$.

Suele presentarse en adultos de 20 a 40 años, con predominio en el sexo femenino en una relación 3:2 ${ }^{10}$, mayormente con enfermedad diseminada ${ }^{2}$, encontrando metástasis hasta en un $27 \%$ de los casos, sobre todo pulmonares ${ }^{8}$. En general las manifestaciones son inespecíficas e incluyen dolor en el cuadrante superior derecho, pérdida de peso, fatiga, ictericia, síndrome de Budd-Chiari e hipertensión portal; algunos casos pueden ser asintomáticos hasta debutar con falla hepática ${ }^{5,11}$.

El abordaje es complejo debido a que generalmente aparece como nódulos que involucran ambos lóbulos hepáticos, indistinguibles por imagen de un carcinoma metastásico ${ }^{3,8}$. Los cambios histológicos presentan una marcada reacción estromal desmosómica con esclerosis densa y pueden imitar a un colangiocarcinoma $^{3}$. El diagnóstico definitivo se realiza por inmunohistoquímica con marcadores positivos para FVIII-RAg, CD 34 y CD $31^{11}$.

El pronóstico sin tratamiento es pobre ${ }^{2}$, con una mortalidad reportada de hasta el $65 \%{ }^{10}$. La escisión 
quirúrgica completa se recomienda para las lesiones resecables $^{5}$. En las lesiones no resecables se han intentado alternativas como quimioembolización transarterial, quimioterapia y radioterapia ${ }^{2,9}$; estas dos últimas con pobre respuesta ${ }^{5}$. El trasplante hepático ortotópico se considera una opción prometedora en lesiones no resecables sin metástasis extrahepáti$\operatorname{cas}^{2,5,9}$ o con falla hepática rápidamente progresiva ${ }^{5}$.

\section{Responsabilidades éticas}

Protección de personas y animales. Los autores declaran que para esta investigación no se han realizado experimentos en seres humanos ni en animales.

Confidencialidad de los datos. Los autores declaran que han seguido los protocolos de su centro de trabajo sobre la publicación de datos de pacientes.

Derecho a la privacidad y consentimiento informado. Los autores han obtenido el consentimiento informado de los pacientes y/o sujetos referidos en el artículo. Este documento obra en poder del autor de correspondencia.

\section{Financiamiento}

Recursos propios de los servicios.

\section{Conflicto de intereses}

Los autores declaran no tener conflicto de intereses.

\section{Bibliografía}

1. Mehrabi A, Kashfi A, Fonouni H, Schemmer P, Schmied BM, Hallscheidt $P$, et al. Primary malignant hepatic epithelioid hemangioendothelioma: a comprehensive review of the literature with emphasis on the surgical therapy. Cancer. 2006;107:2108-21.

2. Remiszewski P, Szczerba E, Kalinowski P, Gierej B, Dudek K, Grodzicki M. Epithelioid hemangioendothelioma of the liver as a rare indication for liver transplantation. World J Gastroenterol. 2014;20:11333.

3. Choi KH, Moon WS. Epithelioid hemangioendothelioma of the liver. Clin Mol Hepatol. 2013;19:315-9.

4. Sweeney WB, Vesoulis Z, Blaum LC. Intravascular bronchioloalveolar tumor: a distinctive surgical and pathological entity. Ann Thorac Surg. 1986;42:702-4.

5. Makhlouf HR, Ishak KG, Goodman ZD. Epithelioid hemangioendothelioma of the liver. A clinicopathologic study of 137 cases. Cancer. 1999;85:562-82.

6. Corrin B, Manners B, Millard M, Weaver L. Histogenesis of the so-called "intravascular bronchioloalveolar tumor." J Pathol 1979;128:163-7.

7. Weldon-Linne CM, Victor TA, Christ ML, Fry WA. Angiogenic nature of "the intravascular bronchioloalveolar tumor" of the lung: an electron microscopic study. Arch Pathol Lab Med 1981;165:174-9.

8. Weiss SW, Enzinger FM. Epithelioid hemangioendothelioma a vascular tumor often mistaken for a carcinoma. Cancer. 1982;50:970-81.

9. Mihaylov V, Kostadinov R, Vasilevski I, Simonova M, Odiseeva E, Vladov N. Liver transplantation for hepatic epithelioid hemangioendothelioma case report and review of the literature. Clin Surg. 2017;2:5.

10. Nudo CG, Yoshida EM, Bain VG, Marleau D, Wong P, Marotta PJ, et al. Liver transplantation for hepatic epithelioid hemangioendothelioma: the Canadian multicentre experience. Can J Gastroenterol. 2008;22:821-4.

11. Wang L-R, Zhou J-M, Zhao Y-M, He H-W, Chai Z-T, Wang M, et al. Clinical experience with primary hepatic epithelioid hemangioendothelioma: retrospective study of 33 patients. World J Surg. 2012;36:2677-83. 